\title{
Generalized Bi-ideal of Ordered Semigroup Related to Intuitionistic Fuzzy Point
}

Hidayat Ullah Khan' ${ }^{*}$, Asghar Khan ${ }^{2}$ and Faiz Muhammad Khan ${ }^{3}$ and Yongjin Li

${ }^{1 *}$ Department of Mathematics, University of Malakand, Khyber Pukhtoonkhwa, Chakdara, Pakistan,

2Department of Mathematics, Abdul Wali Khan University Mardan, Khyber Pukhtoonkhwa, Mardan, Pakistan,

3Department of Mathematics and Statistics, University of Swat, Khyber Pukhtoonkhwa, Swat, Pakistan,

${ }^{4}$ Department of Mathematics, Sun Yat-sen University, Guangzhou, 510275, P. R. China

hidayatullak@yahoo.com, azhar4set@yahoo.com, faiz_zady@yahoo.com, stslyj@mail.sysu.edu.cn

This is an open access article distributed under the Creative Commons Attribution License, which permits unrestricted use, distribution, and reproduction in any medium, provided the original work is properly cited

\section{ARTICLE DETAILS}

Article history:

Received 12 August 2016

Accepted 12 December 2016

Available online 20 January 2017

Keywords:

Intuitionistic fuzzy bi-ideal; $\left(\in, \in \vee q_{k}\right)$-intuitionistic fuzzy biideal; $\quad\left(\in, \in \vee q_{k}\right)$-intuitionistic fuzzy generalized bi-ideals; regular ordered semigroups; left weakly regular ordered semigroups. 2000 Mathematics Subject Classification: 06F05; 20M12; 08A72.

\section{ABSTRACT}

Intuitionistic fuzzy generalized bi-ideals play an important role in the study of ordered semigroups. In this paper, we try obtain more general form of intuitionistic fuzzy generalized bi-ideal of an ordered semigroup. The concept of $\left(\in, \in \vee q_{k}\right)$-intuitionistic fuzzy generalized bi-ideal is introduced and several related properties are investigated. We show that in regular and left weakly regular ordered semigroups the concepts of $\left(\in, \in \vee q_{k}\right)$-intuitionistic fuzzy generalized biideal and $\left(\in, \in \vee q_{k}\right)$-intuitionistic fuzzy bi-ideal coincide.

\section{INTRODUCTION}

A non-empty set $S$ is called an ordered semigroup if $(S, \cdot)$ is a smigroup and $(S, \leq)$ (where $\cdot$ is binary operation on $S$ and $\leq$ is a partial order relation) is a poset such that $a \leq b \rightarrow a \cdot x \leq b \cdot x$ and $x \cdot a \leq x \cdot b$ for all $x \in S$. There are several generalizations of the the fundamental concept of Zadeh's fuzzy subset [1]. One of these generalizations is an intuitionistic fuzzy subset (for short IFSS) [2-5]. An IFSS $A$ in a nonempty set $X$ is an object of the form $A=\left\{\left\langle x, \mu_{A}(x), \gamma_{A}(x)\right\rangle \mid x \in X\right\}$, where $\mu_{A}: X \rightarrow[0,1]$ and $\gamma_{A}: X \rightarrow[0,1]$ respectively are the degrees of membership and non-membership of an element $x \in X$ to the set $A \subseteq X$ and $0 \leq \mu_{A}(x)+\gamma_{A}(x) \leq 1$ for all $x \in X$ [2]. Like Rosenfeld's fuzzy subgroup [6], R. Biswas [7] employed the concept of IFSS in elementary theory of groups and introduced intuitionistic fuzzy subgroup (for short IFSG). Since then, many other researchers employed this concept in; Ring theory [8], in Topological Spaces [9, 10], Semiring [11], Near-ring [12]. The concept of IFSS was first studied by Kim and Jun [13, $14]$ in semigroups and introduced intuitionistic fuzzy (interior) ideals. In addition, Kim and Lee [15] studied intuitionistic fuzzy bi-ideals in semigroups, whereas Jun [[16]] and Khan et al. [17] extended this concept to the structure of ordered semigroups and defined the notion of intuitionistic fuzzy bi-ideals. Jun [17] further investigated that in a regular, left and right simple ordered semigroup, an intuitionistic fuzzy bi-ideal is constant. Moreover, Shabir and Khan [18] introduced intuitionistic fuzzy generalized bi-ideals in ordered semigroups and characterized regular and left weakly regular ordered semigroups in terms of intuitionistic fuzzy generalized bi-ideals. In addition, Khan et al. $[19,20]$ introduced new forms of intuitionistic fuzzy bi-ideals and intuitionistic fuzzy interior ideals in ordered semigroups. In [21], some characterizations of semigroups in terms of intuitionistic fuzzy interior ideals are provided.

In this paper, we define $\left(\in, \in \vee q_{k}\right)$-intuitionistic fuzzy generalized biideals in ordered semigroups, which is a generalization of intuitionistic fuzzy generalized bi-ideals in ordered semigroups given in [18]. We investigate related results of $\left(\in, \in \vee q_{k}\right)$-intuitionistic fuzzy generalized bi-ideals. Further, we prove that in regular and left weakly regular ordered semigroups the concepts of $\left(\in, \in \vee q_{k}\right)$-intuitionistic fuzzy generalized bi-ideal and $\left(\in, \in \vee q_{k}\right)$-intuitionistic fuzzy bi-ideal coincide.

For $\phi \neq A, \phi \neq B \subseteq S$ (ordered semigroup) we denote by ( $A$ ] the subset of $S$ defined as follows:

$$
(A]:=\{t \in S \mid t \leq a \text { for some } a \in A\} .
$$

If $A=\{a\}$, then we write (a] instead of ( $\{a\}]$. The operator ( $]$ is a closure operator, and therefore:

(i) extensive (that is $A \subseteq(A])$

(ii) isotone (that is $A \subseteq B$ implies $(A] \subseteq(B])$ 
(iii) idempotent (that is, $((A]] \subseteq(A]$ and therefore

$$
((A]]=(A]) \text {. }
$$

$A$ is called a subsemigroup of $S$ if $A^{2} \subseteq A$.

$A$ is called a generalized bi-ideal of $S$ if:

(iv) $(\forall a \in S)(\forall b \in A)(a \leq b \rightarrow a \in A)$

(v) $A S A \subseteq A$

$A$ is called a bi-ideal of $S$ if

(vi) $(\forall a \in S)(\forall b \in A)(a \leq b \rightarrow a \in A)$

(vii) $A^{2} \subseteq A$

(viii) $A S A \subseteq A$.

An ordered semigroup $S$ is regular if for every $a \in S$ there exists $x \in S$ such that $a \leq a x a$. An ordered semigroup $S$ is called left (resp. right) regular if for every $a \in S$ there exists $x \in S$, such that $a \leq x a^{2}$ (resp. $a \leq a^{2} x$ ).

Let $t \in(0,1], s \in[0,1)$ such that $0<t+s \leq 1$, then the $\mu_{A}$-level cut and $\gamma_{A}$-level cut of an IFSS $A=\left\langle x, \mu_{A}, \gamma_{A}\right\rangle$ of $S$ are denoted and defined respectively as:

$$
U\left(\mu_{A} ; t\right)=\left\{x \in S \mid \mu_{A}(x) \geq t\right\},
$$

and

$$
L\left(\gamma_{A} ; s\right)=\left\{x \in S \mid \gamma_{A}(x) \leq s\right\} .
$$

The $\left(\mu_{A}, \gamma_{A}\right)$-level cut is denoted and defined as:

$$
C_{(t, s)}(A)=\left\{x \in S \mid \mu_{A}(x) \geq t \text { and } \gamma_{A}(x) \leq s\right\} .
$$

Let $x \in S$ and $t \in(0,1], s \in[0,1)$ such that $0<t+s \leq 1$, then an IFSS of the form

$$
\langle x ;(t, s)\rangle=\left\langle x, x_{t}, 1-x_{1-s}\right\rangle
$$

is called an intuitionistic fuzzy point (for short IFP) in $S$, where $t$ is the degree of membership and $s$ is the degree of non-membership of the IFP $\langle x ;(t, s)\rangle$ and $x$ is the support of the IFP $\langle x ;(t, s)\rangle$.

For a $\phi \neq A \subseteq S$, the characteristic function of $A$ is denoted and defined as:

$$
\chi_{A}=<x, \mu_{\chi_{A}}(x), \gamma_{\chi_{A}}(x)>\text {, }
$$

where

$$
\begin{aligned}
& \mu_{\chi_{A}}(x): S \rightarrow[0,1], x \mapsto \mu_{\chi_{A}}(x):=\left\{\begin{array}{ll}
1 & \text { if } x \in A \\
0 & \text { if } x \notin A
\end{array},\right. \\
& \gamma_{\chi_{A}}(x): S \rightarrow[0,1], x \mapsto \gamma_{\chi_{A}}(x):= \begin{cases}0 & \text { if } x \in A \\
1 & \text { if } x \notin A\end{cases}
\end{aligned}
$$

Definition 1.1 Let $(S, ., \leq)$ be an ordered semigroup and $A=\left\langle x, \mu_{A}, \gamma_{A}\right\rangle$ be an IFSS of $S$. Then $A=\left\langle x, \mu_{A}, \gamma_{A}\right\rangle$ is called $a n$ intuitionistic fuzzy subsemigroup of $S$ if for all $x, y \in S$ :

(a $\left.\mathrm{a}_{1}\right) .\left(\mu_{A}(x y) \geq \min \left\{\mu_{A}(x), \mu_{A}(y)\right\}\right.$

(a2). $\left.\gamma_{A}(x y) \leq \max \left\{\gamma_{A}(x), \gamma_{A}(y)\right\}\right)$

Definition 1.2 An IFSS $A=\left\langle x, \mu_{A}, \gamma_{A}\right\rangle$ of $S$ is called an intuitionistic fuzzy generalized bi-ideal of $S$ if for all $x, y, z \in S$ :

$\left(\mathrm{a}_{3}\right) .(x \leq y) \rightarrow \mu_{A}(x) \geq \mu_{A}(y)$ and $\gamma_{A}(x) \leq \gamma_{A}(y)$

(a) $\mu_{A}(x y z) \geq \min \left\{\mu_{A}(x), \mu_{A}(z)\right\}$ and

$\gamma_{A}(x y z) \leq \max \left\{\gamma_{A}(x), \gamma_{A}(z)\right\}$

Definition 1.3 An intuitionistic fuzzy subsemigroup $A=\left\langle x, \mu_{A}, \gamma_{A}\right\rangle$ of $S$ is called an intuitionistic fuzzy bi-ideal of $S$ if for all $x, y, z \in S$ :

(a5). $(x \leq y) \rightarrow \mu_{A}(x) \geq \mu_{A}(y)$ and $\gamma_{A}(x) \leq \gamma_{A}(y)$

(a) $\mu_{A}(x y z) \geq \min \left\{\mu_{A}(x), \mu_{A}(z)\right\}$ and

$\gamma_{A}(x y z) \leq \max \left\{\gamma_{A}(x), \gamma_{A}(z)\right\}$
Theorem 1.4 An intuitionistic fuzzy subset of $A=\left\langle x, \mu_{A}(x), \gamma_{A}(x)\right\rangle$ of $S$ is an intuitionistic fuzzy generalized bi-ideal of $S$ if and only if

$$
C_{(t, s)}(A)=\left\{x \in S \mid \mu_{A}(x) \geq t \text { and } \gamma_{A}(x) \leq s\right\}(\neq \phi)
$$

is a generalized bi-ideal of $S$, where $t \in(0,1]$ and $s \in[0,1)$.

Theorem 1.5 A non-empty subset $A$ of $S$ is a generalized bi-ideal of $S$ if and only if the characteristic function $\chi_{A}=<x, \mu_{\chi_{A}}, \gamma_{\chi_{A}}>$ of $A$ is an intuitionistic fuzzy generalized bi-ideal of $S$.

\section{MAIN RESULTS}

In this section, we define $\left(\in, \in \vee q_{k}\right)$-intuitionistic fuzzy generalized biideals of an ordered semi group $S$ which is a generalization of intuitionistic fuzzy generalized bi-ideals of $S$, where $k \in[0,1)$. An IFP $\langle x ;(t, s)\rangle$ in said to belong to (resp. quasi-coincident with) an IFSS $A=\left\langle x, \mu_{A}(x), \gamma_{A}(x)>\right.$ of $S$ and denoted as $\langle x ;(t, s)\rangle \in A$ (resp. $\langle x ;(t, s)\rangle q A)$ if $\mu_{A}(x) \geq t$ and $\gamma_{A}(x) \leq s$ (resp. $\mu_{A}(x)+t>1$ and $\left.\gamma_{A}(x)+s<1\right)$. If $\langle x ;(t, s)\rangle \in A$ or $\left.\langle x ;(t, s)\rangle q A\right)$, then we write $\langle x ;(t, s)\rangle \in \vee q A$.

Generalizing this concept quasi-coincident relation of an IFP $\langle x ;(t, s)\rangle$ with an IFSS $A=<x, \mu_{A}(x), \gamma_{A}(x)>$ of $S$. For $k \in[0,1)$ we define $\langle x ;(t, s)\rangle q_{k} A$ whenever $\mu_{A}(x)+t+k>1$ and $\gamma_{A}(x)+s+k<1$. By $\langle x ;(t, s)\rangle \overline{q_{k}} A$ we mean that $\langle x ;(t, s)\rangle q_{k} A$ does not hold. Note that if we define an IFSS $A=<x, \mu_{A}(x), \gamma_{A}(x)>$ of an ordered semigroup $S$ by $\mu_{A}(x) \leq \frac{1-k}{2}$ and $\gamma_{A}(x) \geq \frac{1-k}{2}$ for all $x \in S$, then the set $\left\{\langle x ;(t, s)\rangle \mid\langle x ;(t, s)\rangle \in \wedge q_{k} A\right\}$ is empty. If $\langle x ;(t, s)\rangle \in \wedge q_{k} A$ for some $t \in(0,1]$ and $s \in[0,1)$, then $\mu_{A}(x) \geq t, \mu_{A}(x)+t+k>1$ and $\gamma_{A}(x) \leq s$ and $\gamma_{A}(x)+s+k<1$. From this we can write

and

$$
1<\mu_{A}(x)+t+k \leq \mu_{A}(x)+\mu_{A}(x)+k=2 \mu_{A}(x)+k
$$

$$
1>\gamma_{A}(x)+s+k \geq \gamma_{A}(x)+\gamma_{A}(x)+k=2 \gamma_{A}(x)+k
$$

From this we see that $\mu_{A}(x)>\frac{1-k}{2}$ and $\gamma_{A}(x)<\frac{1-k}{2}$, a contradiction. And hence $\left\{\langle x ;(t, s)\rangle \mid\langle x ;(t, s)\rangle \in \wedge q_{k} A\right\}$ is empty.

Definition 2.1 An intuitionistic fuzzy subset $\left.A=<x, \mu_{A}(x), \gamma_{A}(x)\right\rangle$ of $S$ is called an $\left(\in, \in \vee q_{k}\right)$-intuitionistic fuzzy generalized bi-ideals of $S$, if for all $x, y, z \in S, t, t_{1}, t_{2} \in(0,1]$ and $s, s_{1}, s_{2} \in[0,1)$, satisfies the following conditions:

$\left(\mathrm{b}_{1}\right)$. If $x \leq y$, then $\langle y ;(t, s)\rangle \in A \rightarrow\langle x ;(t, s)\rangle \in \vee q_{k} A$

$\left(\mathrm{b}_{2}\right) .\left\langle x ;\left(t_{1}, s_{1}\right)\right\rangle,\left\langle z ;\left(t_{2}, s_{2}\right)\right\rangle \in A \rightarrow$

$\left\langle x y z ; \min \left\{t_{1}, t_{2}\right\}, \max \left\{s_{1}, s_{2}\right\}\right\rangle \in \vee q_{k} A$

Definition 2.2 [19] An $\left(\in, \in \vee q_{k}\right)$-intuitionistic fuzzy generalized biideals of $S$ is called $\left(\in, \in \vee q_{k}\right)$-intuitionistic fuzzy bi-ideals of $S$ if for all $x, y \in S, t, t_{1}, t_{2} \in(0,1]$ and $s, s_{1}, s_{2} \in[0,1)$, we have

$$
\begin{aligned}
& \left\langle x ;\left(t_{1}, s_{1}\right)\right\rangle,\left\langle y ;\left(t_{2}, s_{2}\right)\right\rangle \in A \rightarrow \\
& \left\langle x y ;\left(\min \left\{t_{1}, t_{2}\right\}, \max \left\{s_{1}, s_{2}\right\}\right)\right\rangle \in \vee q_{k} A .
\end{aligned}
$$

Example 2.3 Consider the ordered semigroup $S=\{a, b, c, d\}$ with multiplication as defined in Table 1 and order relation as given below: 


\section{Table 1}

\begin{tabular}{|l|l|l|l|l|}
\hline$\cdot$ & $a$ & $b$ & $c$ & $d$ \\
\hline$a$ & $a$ & $a$ & $a$ & $a$ \\
\hline$b$ & $a$ & $a$ & $a$ & $a$ \\
\hline$c$ & $a$ & $a$ & $b$ & $a$ \\
\hline$d$ & $a$ & $a$ & $b$ & $b$ \\
\hline
\end{tabular}

$\leq=:\{(a, a),(b, b),(c, c),(a, b)\}$.

Define an IFSS $A=<x, \mu_{A}(x), \gamma_{A}(x)>$ of $S$ as below:

$$
\mu_{A}: S \rightarrow[0,1] \mid x \mapsto \mu_{A}(x)=\left\{\begin{array}{lll}
0.3, & \text { if } & x=a, \\
0.5, & \text { if } & x=b, \\
0.4, & \text { if } & x=c, \\
0.6, & \text { if } & x=d .
\end{array}\right.
$$

and

$$
\gamma_{A}: S \rightarrow[0,1] \mid x \mapsto \gamma_{A}(x)=\left\{\begin{array}{lll}
0.2, & \text { if } & x=a, \\
0.3, & \text { if } & x=b, \\
0.5, & \text { if } & x=c, \\
0.1, & \text { if } & x=d .
\end{array} .\right.
$$

Then it is easy to check that $A$ is an $\left(\in, \in \vee q_{k}\right)$-intuitionistic fuzzy generalized bi-ideals of $S$ for all $k \geq 0.4$.

Remark 1. Clearly an intuitionistic fuzzy generalized bi-ideal is an $\left(\in, \in \vee q_{k}\right)$-intuitionistic fuzzy generalized bi-ideal. However, Example 2.3 shows that $A$ is not an intuitionistic fuzzy generalized bi-ideal. Since

$$
\mu_{A}(b a b)=\mu_{A}(a)=0.3<0.5=\min \left\{\mu_{A}(b), \mu_{A}(b)\right\},
$$

Hence an $\left(\in, \in \vee q_{k}\right)$-intuitionistic fuzzy generalized bi-ideal is not an intuitionistic fuzzy generalized bi-ideal in general.

Theorem 2.4 Let $B$ be a generalized bi-ideal of $S$ and $A$ be an IFSS of $S$ defined as:

and

$$
\mu_{A}(x)\left\{\begin{array}{lll}
\geq \frac{1-k}{2}, & \text { if } & x \in B, \\
=0, & \text { if } & x \notin B,
\end{array}\right.
$$

$$
\gamma_{A}(x)\left\{\begin{array}{ccc}
=0, & \text { if } & x \in B, \\
\leq \frac{1-k}{2}, & \text { if } & x \notin B .
\end{array}\right.
$$

Then $A$ is an $\left(\in, \in \vee q_{k}\right)$-intuitionistic fuzzy generalized bi-ideals of $S$.

Proof. Let $t \in(0,1], s \in[0,1)$ and $x, y \in S$ such that $x \leq y$. If $\langle y ;(t, s)\rangle q A$, then $\mu_{A}(y)+t>1, \gamma_{A}(y)+s<1$ and hence $y \in B$. Then $x \in B$. Indeed: Since $B$ is generalized bi-ideal and $x \leq y \in B$. Hence,

$$
\mu_{A}(x) \geq \frac{1-k}{2} \text { and } \gamma_{A}(x)=0 \leq \frac{1-k}{2} .
$$

We consider the following two cases:

Case I: If $t \leq \frac{1-k}{2}$ and $s \geq \frac{1-k}{2}$, then from the above inequality we have $\mu_{A}(x) \geq t$ and $\gamma_{A}(x) \leq s$ and hence $\langle x ;(t, s)\rangle \in A$.

Case II: If $t>\frac{1-k}{2}$ and $s<\frac{1-k}{2}$, then from the above inequality we have $\mu_{A}(x)+t+k>1$ and $\gamma_{A}(x)+s+k<1$ and so $\langle x ;(t, s)\rangle q_{k} A$. Therefore $\langle x ;(t, s)\rangle \in \vee q_{k} A$.

Next, we let $x, y, z \in S$ and $t_{1}, t_{2} \in(0,1]$ and $s_{1}, s_{2} \in[0,1)$. If $\left\langle x ;\left(t_{1}, s_{1}\right)\right\rangle q A,\left\langle z ;\left(t_{2}, s_{2}\right)\right\rangle q A$, then $\mu_{A}(x)+t_{1}>1, \gamma_{A}(x)+s_{1}<1$ and $\mu_{A}(z)+t_{2}>1, \quad \gamma_{A}(z)+s_{2}<1$ and hence $x, z \in B$. Thus $x y z \in B$. Indeed: Since $B$ is generalized bi-ideal and $x, y, z \in S$ such that $x, z \in B$. Hence

$$
\mu_{A}(x y z) \geq \frac{1-k}{2} \text { and } \gamma_{A}(x y z)=0 \leq \frac{1-k}{2} .
$$

We consider the following two cases: Case III: If $\min \left\{t_{1}, t_{2}\right\} \leq \frac{1-k}{2}$ and $\max \left\{s_{1}, s_{2}\right\} \geq \frac{1-k}{2}$, then from the above inequality we write $\mu_{A}(x y z) \geq \min \left\{t_{1}, t_{2}\right\} \quad$ and $\gamma_{A}(x y z) \leq \max \left\{s_{1}, s_{2}\right\}$ and therefore $\left\langle x y z ;\left(\min \left\{t_{1}, t_{2}\right\}, \max \left\{s_{1}, s_{2}\right\}\right)\right\rangle \in A$.

Case IV: If $\min \left\{t_{1}, t_{2}\right\}>\frac{1-k}{2}$ and $\max \left\{s_{1}, s_{2}\right\}<\frac{1-k}{2}$, then from the above inequality we write $\mu_{A}(x y z)+\min \left\{t_{1}, t_{2}\right\}+k>1$ and $\gamma_{A}(x y z)+\max \left\{s_{1}, s_{2}\right\}+k<1, \quad$ from here we can write $\left\langle x y z ;\left(\min \left\{t_{1}, t_{2}\right\}, \max \left\{s_{1}, s_{2}\right\}\right)\right\rangle q_{k} A$. Hence

$$
\left\langle x y z ;\left(\min \left\{t_{1}, t_{2}\right\}, \max \left\{s_{1}, s_{2}\right\}\right)\right\rangle \in \vee q_{k} A .
$$

Theorem 2.5 Let $B$ be a generalized bi-ideal of $S$ and $A=<x, \mu_{A}(x), \gamma_{A}(x)>$ be an IFSS of $S$ defined by

and

$$
\mu_{A}(x)\left\{\begin{array}{cll}
\geq \frac{1-k}{2}, & \text { if } & x \in B, \\
=0, & \text { if } & x \notin B,
\end{array}\right.
$$

$$
\gamma_{A}(x)\left\{\begin{array}{ccc}
=0, & \text { if } & x \in B, \\
\leq \frac{1-k}{2}, & \text { if } & x \notin B .
\end{array}\right.
$$

Then $A$ is an $\left(\in, \in \vee q_{k}\right)$-intuitionistic fuzzy generalized bi-ideals of $S$. Proof. Let $t \in(0,1], s \in[0,1)$ and $x, y \in S$ such that $x \leq y$. If $\langle y ;(t, s)\rangle \in A$, then $\mu_{A}(y) \geq t, \gamma_{A}(y) \leq s$ and hence $y \in B$. Then $x \in B$. Indeed: Since $B$ is generalized bi-ideal and $x \leq y \in B$. Hence,

$$
\mu_{A}(x) \geq \frac{1-k}{2} \text { and } \gamma_{A}(x)=0<\frac{1-k}{2} .
$$

We consider the following two cases:

Case I: If $t \leq \frac{1-k}{2}$ and $s \geq \frac{1-k}{2}$, then from the above inequality we have $\mu_{A}(x) \geq t$ and $\gamma_{A}(x) \leq s$ and hence $\langle x ;(t, s)\rangle \in A$.

Case II: If $t>\frac{1-k}{2}$ and $s<\frac{1-k}{2}$, then from the above inequality we have $\mu_{A}(x)+t+k>1$ and $\gamma_{A}(x)+s+k<1$ and so $\langle x ;(t, s)\rangle q_{k} A$. Hence $\langle x ;(t, s)\rangle \in \vee q_{k} A$.

Next, we let $x, y, z \in S$ and $t_{1}, t_{2} \in(0,1]$ and $s_{1}, s_{2} \in[0,1)$. If $\left\langle x ;\left(t_{1}, s_{1}\right)\right\rangle \in A,\left\langle z ;\left(t_{2}, s_{2}\right)\right\rangle \in A$, then $\mu_{A}(x) \geq t_{1}, \gamma_{A}(x) \leq s_{1}$ and $\mu_{A}(z) \geq t_{2}, \gamma_{A}(z) \leq s_{2}$ and hence $x, z \in B$. Thus $x y z \in B$. Indeed: Since $B$ is generalized bi-ideal and $x, y, z \in S$ such that $x, z \in B$. Hence

$$
\mu_{A}(x y z) \geq \frac{1-k}{2} \text { and } \quad \gamma_{A}(x y z)=0<\frac{1-k}{2}
$$

We consider the following two cases:

Case III: If $\min \left\{t_{1}, t_{2}\right\} \leq \frac{1-k}{2}$ and $\max \left\{s_{1}, s_{2}\right\} \geq \frac{1-k}{2}$, then from the above inequality we write $\mu_{A}(x y z) \geq \min \left\{t_{1}, t_{2}\right\} \quad$ and $\gamma_{A}(x y z) \leq \max \left\{s_{1}, s_{2}\right\}$. Therefore,

$$
\left\langle x y z ;\left(\min t_{1}, t_{2}, \max \left\{s_{1}, s_{2}\right\}\right)\right\rangle \in A .
$$

Case IV: If $\min \left\{t_{1}, t_{2}\right\}>\frac{1-k}{2}$ and $\max \left\{s_{1}, s_{2}\right\}<\frac{1-k}{2}$, then from the above inequality we write $\mu_{A}(x y z)+\min \left\{t_{1}, t_{2}\right\}+k>1$ and $\gamma_{A}(x y z)+\max \left\{s_{1}, s_{2}\right\}+k<1, \quad$ from here we can write $\left\langle x y z ;\left(\min \left\{t_{1}, t_{2}\right\}, \max \left\{s_{1}, s_{2}\right\}\right)\right\rangle q_{k} A$. This implies

$$
\left\langle x y z ;\left(\min \left\{t_{1}, t_{2}\right\} \max \left\{s_{1}, s_{2}\right\}\right)\right\rangle \in \vee q_{k} A .
$$

Theorem 2.6 An intuitionistic fuzzy subset $A=<x, \mu_{A}(x), \gamma_{A}(x)>$ of $S$ is an $\left(\in, \in \vee q_{k}\right)$-intuitionistic fuzzy generalized bi-ideal of $S$ if and only if the following Coditions (1) and (2) holds simultaniously for all $x, y, z \in S$ : 


$$
\begin{aligned}
& \text { (1). } x \leq y \rightarrow \mu_{A}(x) \geq \min \left\{\mu_{A}(y), \frac{1-k}{2}\right\}, \\
& \gamma_{A}(x) \leq \max \left\{\gamma_{A}(y), \frac{1-k}{2}\right\} \\
& \text { (2). } \mu_{A}(x y z) \geq \min \left\{\mu_{A}(x), \mu_{A}(z), \gamma_{A}(y), \frac{1-k}{2}\right\}, \\
& \gamma_{A}(x y z) \leq \max \left\{\gamma_{A}(x), \gamma_{A}(z), \gamma_{A}(y), \frac{1-k}{2}\right\} .
\end{aligned}
$$

Proof. Let $A$ be an $\left(\in, \in \vee q_{k}\right)$-intuitionistic fuzzy generalized bi-ideal of $S$. Lest there exist $x, y \in S$ such that $x \leq y$ and $\mu_{A}(x)<\min \left\{\mu_{A}(y), \frac{1-k}{2}\right\}, \gamma_{A}(x)>\max \left\{\gamma_{A}(y), \frac{1-k}{2}\right\}$. Then

$$
\begin{aligned}
& \mu_{A}(x)<t \leq \min \left\{\mu_{A}(y), \frac{1-k}{2}\right\}, \\
& \gamma_{A}(x) \geq s>\max \left\{\gamma_{A}(y), \frac{1-k}{2}\right\} .
\end{aligned}
$$

for some $t \in\left(0, \frac{1-k}{2}\right]$ and $s \in\left[\frac{1-k}{2}, 1\right)$. From the above inequality it is clear that $\langle y ;(t, s)\rangle \in A$ but $\langle x ;(t, s)\rangle \in A$ and $\langle x ;(t, s)\rangle \bar{q}_{k} A$. Indeed:

$$
\begin{aligned}
& \mu_{A}(x)+t+k<t+t+k \leq \frac{1-k}{2}+\frac{1-k}{2}+k=1, \\
& \gamma_{A}(x)+s+k \geq s+s+k \geq \frac{1-k}{2}+\frac{1-k}{2}+k=1 .
\end{aligned}
$$

Hence, $\langle x ;(t, s)\rangle \overline{\in \vee q}_{k} A$, a contradiction and therefore we have;

$x \leq y \rightarrow \mu_{A}(x) \geq \min \left\{\mu_{A}(y), \frac{1-k}{2}\right\}, \gamma_{A}(x) \leq \max \left\{\gamma_{A}(y), \frac{1-k}{2}\right\}$, for all $x, y \in S$.

Next we suppose $x, y, z \in S$ such that;

$$
\begin{gathered}
\mu_{A}(x y z)<\min \left\{\mu_{A}(x), \mu_{A}(z), \frac{1-k}{2}\right\}, \\
\gamma_{A}(x)>\max \left\{\gamma_{A}(x), \gamma_{A}(z), \frac{1-k}{2}\right\} .
\end{gathered}
$$

Then there exist $t \in\left(0, \frac{1-k}{2}\right]$ and $s \in\left[\frac{1-k}{2}, 1\right)$ such that

$$
\begin{gathered}
\mu_{A}(x y z)<t \leq \min \left\{\mu_{A}(x), \mu_{A}(z), \frac{1-k}{2}\right\}, \\
\gamma_{A}(x)>s \geq \max \left\{\gamma_{A}(x), \gamma_{A}(z), \frac{1-k}{2}\right\} .
\end{gathered}
$$

From the above inequality we see that $\langle x ;(t, s)\rangle,\langle z ;(t, s)\rangle \in A$ but $\langle x y z ;(t, s)\rangle{\overline{\in \vee q_{k}}} A$, a contradiction. Therefore,

$$
\begin{aligned}
& \mu_{A}(x y z) \geq \min \left\{\mu_{A}(x), \mu_{A}(z), \frac{1-k}{2}\right\}, \\
& \gamma_{A}(x y z) \leq \max \left\{\gamma_{A}(x), \gamma_{A}(z), \frac{1-k}{2}\right\},
\end{aligned}
$$

for all $x, y, z \in S$.

Conversely, let both Condition (1) and Condition (2) hold and $\langle y ;(t, s)\rangle \in A$ for all $t \in(0,1], \quad s \in[0,1)$ and $y \in S$. Then $\mu_{A}(y) \geq t$ and $\gamma_{A}(y) \leq s$ and by Condition (1) we can write,

$$
\begin{aligned}
& \mu_{A}(x) \geq \min \left\{\mu_{A}(y), \frac{1-k}{2}\right\} \geq \min \left\{t, \frac{1-k}{2}\right\}, \\
& \gamma_{A}(x) \leq \max \left\{\gamma_{A}(y), \frac{1-k}{2}\right\} \leq \max \left\{s, \frac{1-k}{2}\right\} .
\end{aligned}
$$

We discuss the following cases:

Case I: If $t>\frac{1-k}{2}$ and $s<\frac{1-k}{2}$, then from the above inequality we have $\mu_{A}(x) \geq \frac{1-k}{2}$ and $\gamma_{A}(x) \leq \frac{1-k}{2}$. This implies,

$$
\begin{aligned}
\mu_{A}(x)+t+k & \geq \frac{1-k}{2}+t+k \\
& >\frac{1-k}{2}+\frac{1-k}{2}+k \\
& =1,
\end{aligned}
$$

and

$$
\begin{aligned}
\gamma_{A}(x)+s+k & \leq \frac{1-k}{2}+s+k \\
& <\frac{1-k}{2}+\frac{1-k}{2}+k \\
& =1 .
\end{aligned}
$$

Hence $\langle x ;(t, s)\rangle q_{k} A$.

Case II: If $t \leq \frac{1-k}{2}$ and $s \geq \frac{1-k}{2}$, then $\mu_{A}(x) \geq t$ and $\gamma_{A}(x) \leq s$ and hence in both the cases we have $\langle x ;(t, s)\rangle \in \vee q_{k} A$.

Next, we consider $\left\langle x ;\left(t_{1}, s_{1}\right)\right\rangle,\left\langle z ;\left(t_{2}, s_{2}\right)\right\rangle \in A$. Then $\mu_{A}(x) \geq t_{1}$, $\gamma_{A}(x) \leq s_{1}$ and $\mu_{A}(z) \geq t_{2}, \gamma_{A}(z) \leq s_{2}$ and from Condition (2) we can write,

$$
\begin{aligned}
& \mu_{A}(x y z) \geq \min \left\{\mu_{A}(x), \mu_{A}(z), \frac{1-k}{2}\right\} \geq \min \left\{t_{1}, t_{2}, \frac{1-k}{2}\right\}, \\
& \gamma_{A}(x y z) \leq \max \left\{\gamma_{A}(x), \gamma_{A}(z), \frac{1-k}{2}\right\} \leq \max \left\{s_{1}, s_{2}, \frac{1-k}{2}\right\} .
\end{aligned}
$$

Here again we discuss two cases as below:

Case III: If $\min \left\{t_{1}, t_{2}\right\}>\frac{1-k}{2}$ and $\max \left\{s_{1}, s_{2}\right\}<\frac{1-k}{2}$, then from above inequality we have $\mu_{A}(x y z) \geq \frac{1-k}{2}, \gamma_{A}(x y z) \leq \frac{1-k}{2}$. This implies,

$$
\begin{aligned}
\mu_{A}(x y z)+\min \left\{t_{1}, t_{2}\right\}+k & \geq \frac{1-k}{2}+\min \left\{t_{1}, t_{2}\right\}+k \\
& >\frac{1-k}{2}+\frac{1-k}{2}+k=1, \\
\gamma_{A}(x y z)+\max \left\{s_{1}, s_{2}\right\}+k & \leq \frac{1-k}{2}+\max \left\{s_{1}, s_{2}\right\}+k \\
& <\frac{1-k}{2}+\frac{1-k}{2}+k=1 .
\end{aligned}
$$

Hence $\left\langle x y z ;\left(\min \left\{t_{1}, t_{2}\right\}, \max \left\{s_{1}, s_{2}\right\}\right)\right\rangle q_{k} A$.

Case IV: If $\min \left\{t_{1}, t_{2}\right\} \leq \frac{1-k}{2}$ and $\max \left\{s_{1}, s_{2}\right\} \geq \frac{1-k}{2}$, then again from above inequality we have $\mu_{A}(x y z) \geq \min \left\{t_{1}, t_{2}\right\} \quad$ and $\gamma_{A}(x y z) \leq \max \left\{s_{1}, s_{2}\right\}$. Hence,

$$
\left\langle x y z ;\left(\min \left\{t_{1}, t_{2}\right\}, \max \left\{s_{1}, s_{2}\right\}\right)\right\rangle \in \vee q_{k} A .
$$

Consequently $A$ is an $\left(\in, \in \vee q_{k}\right)$-intuitionistic fuzzy generalized biideal of $S$.

Theorem 2.7 Let $A$ be an IFSS of $S$ and $C_{(t, s)}(A) \neq \phi$ for all $t \in\left(0, \frac{1-k}{2}\right]$ and $s \in\left(\frac{1-k}{2}, 1\right)$. Then the following are equivalent:

(1). $A$ is an $\left(\in, \in \vee q_{k}\right)$-intuitionistic fuzzy generalized bi-ideal.

(2). $C_{(t, s)}(A)$ is a generalized bi-ideal.

Proof. (1) $\Rightarrow$ (2): Let $t \in\left(0, \frac{1-k}{2}\right], s \in\left(\frac{1-k}{2}, 1\right)$ and $x, y \in S$ such that $x \leq y \in C_{(t, s)}(A)$. Then $\mu_{A}(y) \geq t$ and $\gamma_{A}(y) \leq s$. By hypothesis,

$$
\begin{aligned}
& \mu_{A}(x) \geq \min \left\{\mu_{A}(y), \frac{1-k}{2}\right\} \geq \min \left\{t, \frac{1-k}{2}\right\}=t, \\
& \gamma_{A}(x) \leq \max \left\{\gamma_{A}(y), \frac{1-k}{2}\right\} \leq \max \left\{s, \frac{1-k}{2}\right\}=s .
\end{aligned}
$$

These inequalities implies $x \in C_{(t, s)}(A)$.

If $x, y, z \in S$ such that $x, z \in C_{(t, s)}(A)$, then $\mu_{A}(x) \geq t, \gamma_{A}(x) \leq s$ and $\mu_{A}(z) \geq t, \gamma_{A}(z) \leq s$. By Condition (1) we have,

$$
\begin{aligned}
& \mu_{A}(x y z) \geq \min \left\{\mu_{A}(x), \mu_{A}(z), \frac{1-k}{2}\right\} \geq \min \left\{t, t, \frac{1-k}{2}\right\}=t, \\
& \gamma_{A}(x y z) \leq \max \left\{\gamma_{A}(x), \gamma_{A}(z), \frac{1-k}{2}\right\} \leq \max \left\{s, s, \frac{1-k}{2}\right\}=s .
\end{aligned}
$$

From the above inequalities we write, $x y z \in C_{(t, s)}(A)$. Hence, $C_{(t, s)}(A)$ is a generalized bi-ideal of $S$ for all $t \in\left(0, \frac{1-k}{2}\right]$ and $s \in\left(\frac{1-k}{2}, 1\right)$.

(2) $\Rightarrow$ (1): Let $C_{(t, s)}(A)$ is a generalized bi-ideal and suppose on contrary that Condition (1) does not hold. Then there exist $x, y \in S$ with $x \leq y$ such that Then

$$
\mu_{A}(x)<\min \left\{\mu_{A}(y), \frac{1-k}{2}\right\} \text { and } \gamma_{A}(x)>\max \left\{\gamma_{A}(y), \frac{1-k}{2}\right\} .
$$

$\mu_{A}(x)<t \leq \min \left\{\mu_{A}(y), \frac{1-k}{2}\right\}$ and $\gamma_{A}(x)>s \geq \max \left\{\gamma_{A}(y), \frac{1-k}{2}\right\}$ for some $t \in\left(0, \frac{1-k}{2}\right]$ and $s \in\left(\frac{1-k}{2}, 1\right)$. This implies $y \in C_{(t, s)}(A)$ but $\overline{x \in C_{(t, s)}}(A)$, a contradiction. Thus $x \leq y \rightarrow \mu_{A}(x) \geq \min \left\{\mu_{A}(y), \frac{1-k}{2}\right\}$ and $\gamma_{A}(x) \leq \max \left\{\gamma_{A}(y), \frac{1-k}{2}\right\}$ for all $x, y \in S$. If there exist $x, y, z \in S$ such that

$$
\begin{gathered}
\mu_{A}(x y z)<\min \left\{\mu_{A}(x), \mu_{A}(z), \frac{1-k}{2}\right\}, \\
\gamma_{A}(x y z)>\max \left\{\gamma_{A}(x), \gamma_{A}(z), \frac{1-k}{2}\right\} .
\end{gathered}
$$


Then for some $t \in\left(0, \frac{1-k}{2}\right]$ and $s \in\left(\frac{1-k}{2}, 1\right)$ we have

$$
\begin{aligned}
& \mu_{A}(x y z)<t \leq \min \left\{\mu_{A}(x), \mu_{A}(z), \frac{1-k}{2}\right\}, \\
& \gamma_{A}(x y z)>s \geq \max \left\{\gamma_{A}(x), \gamma_{A}(z), \frac{1-k}{2}\right\} .
\end{aligned}
$$

The above inequality implies $x, z \in C_{(t, s)}(A)$ but $x y z \in \bar{C}_{(t, s)}(A)$, a contradiction. Therefore

$$
\begin{aligned}
& \mu_{A}(x y z) \geq \min \left\{\mu_{A}(x), \mu_{A}(z), \frac{1-k}{2}\right\}, \\
& \gamma_{A}(x y z) \leq \max \left\{\gamma_{A}(x), \gamma_{A}(z), \frac{1-k}{2}\right\}
\end{aligned}
$$

for all $x, y, z \in S$. Hence by Theorem (2.6), $A$ is an $\left(\in, \in \vee q_{k}\right)$ intuitionistic fuzzy generalized bi-ideal of $S$.

Proposition 2.8. Every $\left(\in, \in \vee q_{k}\right)$-intuitionistic fuzzy generalized biideal of a regular ordered semigroup $S$ is an $\left(\in, \in \vee q_{k}\right)$-intuitionistic fuzzy bi-ideal of $S$.

Proof. Let $a, b \in S$ and $A$ an $\left(\in, \in \vee q_{k}\right)$-intuitionistic fuzzy generalized bi-ideal $S$. Since $S$ is regular, therefore there exist $x \in S$ such that $b \leq b x b$ and so

$$
\begin{aligned}
\mu_{A}(a b) & \geq \mu_{A}(a(b x b)) \\
& =\mu_{A}(a(b x) b) \\
& \geq \min \left\{\mu_{A}(a), \mu_{A}(b), \frac{1-k}{2}\right\}, \\
\gamma_{A}(a b) & \leq \gamma_{A}(a(b x b)) \\
& =\gamma_{A}(a(b x) b) \\
& \leq \max \left\{\gamma_{A}(a), \gamma_{A}(b), \frac{1-k}{2}\right\} .
\end{aligned}
$$

This shows that $A$ is an $\left(\in, \in \vee q_{k}\right)$-intuitionistic fuzzy subsemigroup of $S$. Thus $A$ is an $\left(\in, \in \vee q_{k}\right)$-intuitionistic fuzzy bi-ideal of $S$.

Proposition 2.9 Every $\left(\in, \in \vee q_{k}\right)$-intuitionistic fuzzy generalized biideal of a left weakly regular ordered semigroup $S$ is an $\left(\in, \in \vee q_{k}\right)$. intuitionistic fuzzy bi-ideal of $S$.

Proof. Let $a, b \in S$ and $A$ an $\left(\in, \in \vee q_{k}\right)$-intuitionistic fuzzy generalized bi-ideal $S$. Since $S$ is left weakly regular, therefore there exist $x, y \in S$ such that $b \leq x b y b$ and therefore,

$$
\begin{aligned}
\mu_{A}(a b) & \geq \mu_{A}(a(x b y b)) \\
& =\mu_{A}(a(x b y) b) \\
& \geq \min \left\{\mu_{A}(a), \mu_{A}(b), \frac{1-k}{2}\right\}, \\
\gamma_{A}(a b) & \leq \gamma_{A}(a(x b y b)) \\
& =\gamma_{A}(a(x b y) b) \\
& \leq \max \left\{\gamma_{A}(a), \gamma_{A}(b), \frac{1-k}{2}\right\} .
\end{aligned}
$$

Thus $A$ is an $\left(\in, \in \vee q_{k}\right)$-intuitionistic fuzzy bi-ideal of $S$.

From Remark 1 we observe that an intuitionistic fuzzy generalized biideal is an $\left(\in, \in \vee q_{k}\right)$-intuitionistic fuzzy generalized bi-ideal but conversely it is not true in general. In the next result we show that if for an IFSS $A$ of an ordered semigroup $S$ we have $\mu_{A}(x) \leq \frac{1-k}{2}$ and $\gamma_{A}(x) \geq \frac{1-k}{2}$ for all $x \in S$, then every $\left(\in, \in \vee q_{k}\right)$-intuitionistic fuzzy generalized bi-ideal is an intuitionistic fuzzy generalized bi-ideal.

Theorem 2.10 Let $A=<x, \mu_{A}(x), \gamma_{A}(x)>$ be an $\left(\in, \in \vee q_{k}\right)$ intuitionistic fuzzy generalized bi-ideal of $S$ such that $\mu_{A}(x) \leq \frac{1-k}{2}$ and $\gamma_{A}(x) \geq \frac{1-k}{2}$ for all $x \in S$. Then $A$ is an intuitionistic fuzzy generalized bi-ideal.

Proof. Let $x, y \in S$ such that $x \leq y$. Then by Theorem 2.6 (1) we have

$$
\begin{aligned}
& \mu_{A}(x) \geq \min \left\{\mu_{A}(y), \frac{1-k}{2}\right\}=\mu_{A}(y), \\
& \gamma_{A}(x) \leq \max \left\{\gamma_{A}(y), \frac{1-k}{2}\right\}=\gamma_{A}(y) .
\end{aligned}
$$

Let $x, y, z \in S$. Then by Theorem 2.6 (2)

$$
\begin{aligned}
& \mu_{A}(x y z) \geq \min \left\{\mu_{A}(x), \mu_{A}(z), \frac{1-k}{2}\right\}=\min \left\{\mu_{A}(x), \mu_{A}(z)\right\}, \\
& \gamma_{A}(x y z) \leq \max \left\{\gamma_{A}(x), \gamma_{A}(z), \frac{1-k}{2}\right\}=\max \left\{\gamma_{A}(x), \gamma_{A}(z)\right\} .
\end{aligned}
$$

Hence $A$ is an intuitionistic fuzzy generalized bi-ideal.

Proposition 2.11 If $A=<x, \mu_{A}(x), \gamma_{A}(x)>$ is a non-zero $\left(\in, \in \vee q_{k}\right)$ -intuitionistic fuzzy generalized bi-ideal of $S$. Then the set

$$
A_{\frac{1-k}{2}}=\left\{x \in S: \mu_{A}(x) \geq \frac{1-k}{2} \text { and } \gamma_{A}(x) \leq \frac{1-k}{2}\right\}
$$

is a generalized bi-ideal of $S$.

Proof. Consider $A$ is an $\left(\in, \in \vee q_{k}\right)$-intuitionistic fuzzy generalized biideal of $S$. Let $a, b \in S$ such that $a \leq b \in A_{\frac{1-k}{2}}$, then $\mu_{A}(b) \geq \frac{1-k}{2}$ and $\gamma_{A}(b) \leq \frac{1-k}{2}$. By hypothesis we have,

$$
\begin{aligned}
\mu_{A}(a) & \geq \min \left\{\mu_{A}(b), \frac{1-k}{2}\right\} \\
& \geq \frac{1-k}{2}\left(\text { since } \mu_{A}(b) \geq \frac{1-k}{2}\right), \\
\gamma_{A}(a) & \leq \max \left\{\gamma_{A}(b), \frac{1-k}{2}\right\} \\
& \leq \frac{1-k}{2}\left(\text { since } \gamma_{A}(b) \leq \frac{1-k}{2}\right) .
\end{aligned}
$$

This implies $a \in A_{\frac{1-k}{2}}$.

Next suppose that $a, b, c \in S$ such that $a, c \in A_{\frac{1-k}{2}}$, then $\mu_{A}(a) \geq \frac{1-k}{2}$, $\gamma_{A}(a) \leq \frac{1-k}{2}$ and $\mu_{A}(c) \geq \frac{1-k}{2}, \gamma_{A}(c) \leq \frac{1-k}{2}$. By hypothesis,

$$
\begin{aligned}
\mu_{A}(a b c) & \geq \min \left\{\mu_{A}(a), \mu_{A}(c), \frac{1-k}{2}\right\} \\
& \geq \frac{1-k}{2}\left(\text { since } \mu_{A}(a) \geq \frac{1-k}{2}, \mu_{A}(c) \geq \frac{1-k}{2}\right), \\
\gamma_{A}(a b c) & \leq \max \left\{\gamma_{A}(a), \gamma_{A}(c), \frac{1-k}{2}\right\} \\
& \leq \frac{1-k}{2}\left(\text { since } \gamma_{A}(a) \leq \frac{1-k}{2}, \gamma_{A}(c) \leq \frac{1-k}{2}\right) .
\end{aligned}
$$

So $a b c \in A_{\frac{1-k}{2}}$ and consequently $A_{\frac{1-k}{2}}$ is a generalized bi-ideal of $S$.

Theorem 2.12 A non-empty subset $A$ of $S$ is a generalized bi-ideal of $S$ if and only if the characteristic function $\chi_{A}=<x, \mu_{\chi_{A}}, \gamma_{\chi_{A}}>$ of $A$ is an $\left(\in, \in \vee q_{k}\right)$-intuitionistic fuzzy generalized bi-ideal of $S$.

Proof. Let $A$ is a generalized bi-ideal of $S$ and $x, y \in S$ with $x \leq y$. If $y \in A$, then $x \in A$ (since $A$ is a generalized bi-ideal of $S$ ) and hence,

$$
\begin{aligned}
& \mu_{\chi_{A}}(y)=1 \text { and } \gamma_{x_{A}}(y)=0, \\
& \mu_{\chi_{A}}(x)=1 \text { and } \gamma_{\chi_{A}}(x)=0 .
\end{aligned}
$$

therefore,

$$
\begin{aligned}
& \min \left\{\mu_{\chi_{A}}(y), \frac{1-k}{2}\right\}=\min \left\{1, \frac{1-k}{2}\right\}=\frac{1-k}{2}<1=\mu_{\chi_{A}}(x), \\
& \max \left\{\gamma_{x_{A}}(y), \frac{1-k}{2}\right\}=\max \left\{0, \frac{1-k}{2}\right\}=\frac{1-k}{2}>0=\gamma_{x_{A}}(x)
\end{aligned}
$$

If $y \notin A$ and $x \in A$, then

$$
\begin{aligned}
& \mu_{\chi_{A}}(y)=0 \text { and } \gamma_{\chi_{A}}(y)=1, \\
& \mu_{\chi_{A}}(x)=1 \text { and } \gamma_{\chi_{A}}(x)=0 .
\end{aligned}
$$

Therefore,

$$
\begin{aligned}
& \min \left\{\mu_{\chi_{A}}(y), \frac{1-k}{2}\right\}=\min \left\{0, \frac{1-k}{2}\right\}=0<1=\mu_{\chi_{A}}(x), \\
& \max \left\{\gamma_{\chi_{A}}(y), \frac{1-k}{2}\right\}=\max \left\{1, \frac{1-k}{2}\right\}=1>0=\gamma_{\chi_{A}}(x) .
\end{aligned}
$$

If $y \notin A$ and $x \notin A$, then

$$
\begin{aligned}
& \mu_{\chi_{A}}(y)=0 \text { and } \gamma_{\chi_{A}}(y)=1, \\
& \mu_{\chi_{A}}(x)=0 \text { and } \gamma_{\chi_{A}}(x)=1 .
\end{aligned}
$$

Hence,

$$
\begin{aligned}
& \min \left\{\mu_{\chi_{A}}(y), \frac{1-k}{2}\right\}=\min \left\{0, \frac{1-k}{2}\right\}=0=\mu_{\chi_{A}}(x), \\
& \max \left\{\gamma_{\chi_{A}}(y), \frac{1-k}{2}\right\}=\max \left\{1, \frac{1-k}{2}\right\}=1=\gamma_{\chi_{A}}(x) .
\end{aligned}
$$

Hence in all the above cases we have,

$$
\mu_{\chi_{A}}(x) \geq \min \left\{\mu_{\chi_{A}}(y), \frac{1-k}{2}\right\} \text { and } \gamma_{x_{A}}(x) \leq \max \left\{\gamma_{x_{A}}(y), \frac{1-k}{2}\right\} \text {. }
$$

Next we suppose $x, y, z \in S$ such that $x, z \in A$, then $x y z \in A$ (since 
$A$ is a generalized bi-ideal of $S$ ). Hence,

$$
\begin{gathered}
\mu_{\chi_{A}}(x)=1 \text { and } \gamma_{\chi_{A}}(x)=0, \\
\mu_{\chi_{A}}(z)=1 \text { and } \gamma_{\chi_{A}}(z)=0, \\
\mu_{\chi_{A}}(x y z)=1 \text { and } \gamma_{\chi_{A}}(x y z)=0 .
\end{gathered}
$$

Then,

$$
\begin{aligned}
& \min \left\{\mu_{\chi_{A}}(x), \mu_{\chi_{A}}(z), \frac{1-k}{2}\right\}=\min \left\{1,1, \frac{1-k}{2}\right\}=\frac{1-k}{2}<1=\mu_{\chi_{A}}(x y z), \\
& \max \left\{\gamma_{\chi_{A}}(x), \gamma_{\chi_{A}}(z), \frac{1-k}{2}\right\}=\max \left\{0,0, \frac{1-k}{2}\right\}=\frac{1-k}{2}>0=\gamma_{\chi_{A}}(x y z) .
\end{aligned}
$$

If $x, z \notin A$ and $x y z \in A$, then,

$$
\begin{gathered}
\mu_{\chi_{A}}(x)=0 \text { and } \gamma_{\chi_{A}}(x)=1, \\
\mu_{\chi_{A}}(z)=0 \text { and } \gamma_{\chi_{A}}(z)=1, \\
\mu_{\chi_{A}}(x y z)=1 \text { and } \gamma_{x_{A}}(x y z)=0,
\end{gathered}
$$

Therefore,

$$
\begin{aligned}
& \min \left\{\mu_{\chi_{A}}(x), \mu_{\chi_{A}}(z), \frac{1-k}{2}\right\}=\min \left\{0,0, \frac{1-k}{2}\right\}=0<1=\mu_{\chi_{A}}(x y z), \\
& \max \left\{\gamma_{\chi_{A}}(x), \gamma_{\chi_{A}}(z), \frac{1-k}{2}\right\}=\max \left\{1,1, \frac{1-k}{2}\right\}=1>0=\gamma_{\chi_{A}}(x y z) .
\end{aligned}
$$

If $x, z \notin A$ and $x y z \notin A$, then

$$
\begin{gathered}
\mu_{\chi_{A}}(x)=0 \text { and } \gamma_{\chi_{A}}(x)=1, \\
\mu_{\chi_{A}}(z)=0 \text { and } \gamma_{x_{A}}(z)=1, \\
\mu_{\chi_{A}}(x y z)=0 \text { and } \gamma_{x_{A}}(x y z)=1 .
\end{gathered}
$$

Hence

$$
\begin{gathered}
\min \left\{\mu_{\chi_{A}}(x), \mu_{\chi_{A}}(z), \frac{1-k}{2}\right\}=\min \left\{0,0, \frac{1-k}{2}\right\}=0=\mu_{\chi_{A}}(x y z), \\
\max \left\{\gamma_{\chi_{A}}(x), \gamma_{\chi_{A}}(z), \frac{1-k}{2}\right\}=\max \left\{1,1, \frac{1-k}{2}\right\}=1=\gamma_{\chi_{A}}(x y z) .
\end{gathered}
$$

From the above discussion we have,

$$
\begin{gathered}
\mu_{\chi_{A}}(x y z) \geq \min \left\{\mu_{\chi_{A}}(x), \mu_{\chi_{A}}(z), \frac{1-k}{2}\right\}, \\
\gamma_{\chi_{A}}(x y z) \leq \max \left\{\gamma_{\chi_{A}}(x), \gamma_{\chi_{A}}(z), \frac{1-k}{2}\right\}=\max \left\{1,1, \frac{1-k}{2}\right\}=1=.
\end{gathered}
$$

Consequently $\chi_{A}$ is an $\left(\in, \in \vee q_{k}\right)$-intuitionistic fuzzy generalized biideal of $S$.

Conversely, let $\chi_{A}$ is an $\left(\in, \in \vee q_{k}\right)$-intuitionistic fuzzy generalized biideal of $S$ and $x, y \in S$ such that $x \leq y \in A$. Then $\mu_{\chi_{\mathrm{A}}}(y)=1$ and $\gamma_{\chi_{A}}(y)=0$. By hypothesis,

$$
\begin{aligned}
& \mu_{\chi_{A}}(x) \geq \min \left\{\mu_{\chi_{A}}(y), \frac{1-k}{2}\right\}=\min \left\{1, \frac{1-k}{2}\right\}=\frac{1-k}{2}, \\
& \gamma_{x_{A}}(x) \leq \max \left\{\gamma_{x_{A}}(y), \frac{1-k}{2}\right\}=\max \left\{0, \frac{1-k}{2}\right\}=\frac{1-k}{2},
\end{aligned}
$$

hence $x \in A$.

Next we suppose $x, y, z \in S$ such that $x, z \in A$, then

$$
\mu_{\chi_{A}}(x)=1=\mu_{\chi_{A}}(z) \text { and } \gamma_{x_{A}}(x)=0=\gamma_{x_{A}}(z) \text {. }
$$

By hypothesis we have,

$$
\begin{aligned}
& \mu_{\chi_{A}}(x y z) \geq \min \left\{\mu_{\chi_{A}}(x), \mu_{\chi_{A}}(z), \frac{1-k}{2}\right\}=\min \left\{1,1, \frac{1-k}{2}\right\}=\frac{1-k}{2}, \\
& \gamma_{\chi_{A}}(x y z) \leq \max \left\{\gamma_{\chi_{A}}(x), \gamma_{\chi_{A}}(z), \frac{1-k}{2}\right\}=\max \left\{0,0, \frac{1-k}{2}\right\}=\frac{1-k}{2} .
\end{aligned}
$$

This shows that $x y z \in A$. Hence $A$ is a generalized bi-ideal of $S$.

\section{Conclusion}

An intuitionistic fuzzy subset (for short IFSS) is one of the generalizations of Zadeh's fuzzy subset. Intuitionistic fuzzy sets entertain every element by attributing both a grade of membership $t \in(0,1]$ and a nonmembership $s \in[0,1)$ such that $0<t+s \leq 1$. Further, ordered semigroups play a key role in the field of mathematics with a large number of applications in different areas like coding theory, automata theory and sequential machine, language theory and formal grammar and neural networks. We employed the concept of IFSS in ordered semigroups and introduced a new generalization of generalization of intuitionistic fuzzy generalizd bi-ideals. This new generalization is called $\left(\in, \in \vee q_{k}\right)$ -intuitionistic fuzzy generalizd bi-ideals. We invistigate related results of $\left(\in, \in \vee q_{k}\right)$-intuitionistic fuzzy generalizd bi-ideals. Further, we prove that in regular and left weakly regular ordered semigroups the concepts of $\left(\in, \in \vee q_{k}\right)$-intuitionistic fuzzy generalizd bi-ideal and $\left(\in, \in \vee q_{k}\right)$. intuitionistic fuzzy bi-ideal coincide.

Acknowledgement. This work was supported by the National Natural Science Foundation of China (11571378) and University of Malakand. 


\section{References}

[1] Zadeh, L. A., Fuzzy sets, Information and control, 8 338-3353.

[2] Atanassov, K. T., Intuitionistic fuzzy sets, in: V. Sgurev, ITKR's Session, Sofia, (Central Sci. and Techn. Library, Academy of Science), June 1983.

[3] Atanassov, K. T., Intuitionistic fuzzy sets, theory and applications, Studies in Fuzziness and Soft Computing Sofia, Heidelberg, Physical-Verlag 35, (1999).

[4] Atanassov, K. T., Intuitionistic fuzzy sets, Fuzzy Sets and Systems, 20 (1986), 87-96.

[5] Atanassov, K. T., New operations defined over the intuitionistic fuzzy sets, Fuzzy Sets and Systems, 61 137-142.

[6] Rosenfeld, A., Fuzzy Groups, Journal of Mathematical and Application, 35 (1971), 512-517.

[7] Biswas, R., Intuitionistic fuzzy subgroups, Math. Forum., 37-46.

[8] Banerjee, B. and Basnet, D. K., Intuitionistic fuzzy and ideals, J. Fuzzy Math., 11 (1) (2003), 139- 155.

[9] Coker, D., An introduction to intuitionistic fuzzy spaces, Fuzzy Sets and Systems, (1996), 293- 303.

Analysis

10 (1989),

subrings

topological

[10] Coker, D. and Haydar Es, A., On fuzzy compactness in intuitionistic fuzzy topological spaces, J. Fuzzy Math., 3 (1995) 899-909.

[11] Hur, K., Jang, S. Y. and Lee, K. C., Intuitionistic fuzzy $\quad$ weak congruences on a semiring, Internantional journal of fuzzy logic and intelligent systems, 6 (4) (2006), 321- 330.

[12] Hur, K., Jang, S. Y. and Lee, K. C., Intuitionistic fuzzy $\quad$ weak congruences on a near-ring module, J. Korea Soc. Math. Educ. Ser B: Pure Appl. Math, 13 (3) (2006), 167-187.

[13] Kim, K. H. and Jun, Y. B., Intuitionistic fuzzy ideals of semigroups, Indian journal of pure and Applied Mathematics, 33 (4) (2002), 443-449.

[14] Kim, K. H. and Jun, Y. B., Intuitionistic fuzzy interior ideals of semigroups, Int. J. Math. Sci., 27 (5) (2001), $\quad$ 261-267.

[15] Kim, K. H. and Lee, J. G., On Intuitionistic Fuzzy Bi- Ideals of Semigroups, Turk J Math, 29 (2005), 201-210.

[16] Jun, Y. B., Intuitionistic Fuzzy Bi-ideals of Ordered Semigroups, Kyungpook Math. J., 45 (2005), 527-537.

[17] Khan, A., Jun, Y. B. and Shabir, M., Ordered characterized by their intuitionistic fuzzy bi-

semigroups of fuzzy systems, 7 (2) (2010), 55-69.

ideals, Iranian journal

[18] Shabir, M. and Khan, A., Ordered Semigroups characterizations by Their Intuitionistic Fuzzy Generalized Bi-ideals, Fuzzy System and Mathematics, $\quad 23$ (2009).

[19] Khan, A., Davvaz, B., Sarmin, N. H. and Khan, H., Inequalities and Applications 2013,

(http://www.journalofinequalitiesandapplications.com/content/2013/ 1/397)

[20] Khan, H., Sarmin, N. H., Khan, A. and Khan, F. M., $\quad$ New types of intuitionistic fuzzy interior ideals of ordered semigroups, Annals of Fuzzy Mathematics and $\quad$ Informatics, 6 (3) (2013), 495-519.

[21] Khan, H., Sarmin, N. H., Khan, A. and Khan, F. M., $\quad$ Some 